\title{
Logic-based reasoning support for SBVR
}

\author{
Dmitry Solomakhin ${ }^{1)}$, Enrico Franconi ${ }^{2)}$, and Alessandro Mosca ${ }^{2)}$ \\ Free University of Bozen-Bolzano, Italy \\ Piazza Domenicani 3, 39100 Bolzano, Italy \\ ${ }^{1)}$ Dmitry . Solomakhin@stud-inf . unibz .it, ${ }^{2)}$ [surname] @inf . unibz . it
}

\begin{abstract}
Automated support to enterprise modeling has increasingly become a subject of interest for organizations seeking solutions for storage, distribution and analysis of knowledge about business processes. This interest has recently resulted in approving the standard for specifying Semantics of Business Vocabulary and Business Rules (SBVR). Despite the existence of formally grounded notations, up to now SBVR still lacks a sound and consistent logical formalization which would allow developing automated solutions able to check the consistency of a set of business rules. This work reports on the attempt to provide logical foundations for SBVR by the means of defining a specific first-order deonticalethic logic (FODAL). The connections of FODAL with the modal logic $\boldsymbol{Q K}$ and the description logic $\mathcal{A L C} \mathcal{L I}$ have been investigated and, on top of the obtained theoretical results, a special tool providing automated support for consistency checks of a set of $\mathcal{A L C} \mathcal{Q I}$-expressible deontic and alethic business rules has been implemented.
\end{abstract}

\section{Introduction}

Automated support to enterprise modeling has increasingly become a subject of interest for organizations seeking solutions for storage, distribution and analysis of knowledge about business processes. One of the most common approaches for describing business and the information used by that business is the rule-based approach 4, which was adopted by the Object Management Group (OMG) for a standard for specifying business objects and rules. The Semantics of Business Vocabulary and Business Rules (SBVR) [19] standard provides means for describing the structure of the meaning of rules, so called "semantic formulation", expressed in one of the intuitive notations, including the natural language that business people use [2] and Object-Role Modeling (ORM2) diagrams [8]. ORM2 has recently become widely used as conceptual modeling approach combining both formal, textual specification language and graphical modeling language 9]. It consists in identifying and articulating the rules that define the structure (alethic) and control the operation (deontic) of an enterprise [18. The main expectation from automated solutions built upon this approach is the ability to automatically determine consistency of business rules in a business model, so that they can be further exploited to build information systems and relational databases that are coherent with the intended domain business logic. 
Several attempts have been made so far in order to provide a logical formalization for structural and operational rules in SBVR and its notations. The most significant related work includes several formalizations of the purely structural fragment of ORM2, including translation to first-order predicate logic (FOL) [10] and some description logics (DL), e.g. [14] and [12. However, none of the existing approaches enables consistency checks for a combined set of possibly interacting alethic and deontic business rules.

In this paper we define a multimodal first-order deontic-alethic logic (FO$D A L$ ) with sound and complete axiomatization that captures the desired semantics of and interaction between business rules. We then report on the logical properties of such formalization and its connections with the modal logic $\boldsymbol{Q} \boldsymbol{K}$ and the description logic $\mathcal{A L C} \mathcal{Q I}$. Finally we present the tool which provides

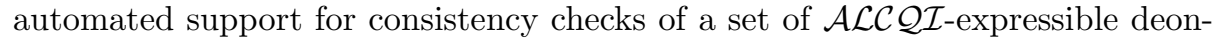
tic and alethic ORM2 constraints. Additionally, it implements the translation of aforementioned class of ORM2 constraints into an OWL2 ontology.

The rest of the paper is organized as follows. In the second section an overview of the SBVR standard and its ORM2 notation is given. Third section describes the proposed logical formalization in terms of first-order deontic-alethic logic (FODAL) along with its syntax, semantics and complete and sound axiomatization. Next two paragraphs are devoted to modeling SBVR rules with FODAL and checking their consistency with the help of this logic, while in the sixth section a connection with standard modal logic is introduced. Finally, the last paragraph describes the tool developed to provide automated support for consistency checks together with translation to OWL2.

\section{SBVR Overview}

A core idea of business rules formally supported by SBVR is the following [19]: "Rules build on facts, and facts build on concepts as expressed by terms. Terms express business concepts; facts make assertions about these concepts; rules constrain and support these facts". The notions of terms and facts of this "business rules mantra" correspond to SBVR noun concepts and verb concepts (or fact types) respectively.

Noun and verb concepts. According to the SBVR 1.0 specification [19] a noun concept is defined as a "concept that is the meaning of a noun or noun phrase". It has several subtypes: object type, individual concept and fact type role. An object type is defined as "noun concept that classifies things on the basis of their common properties", while individual concept is "a concept that corresponds to only one object [thing]". A role is a "noun concept that corresponds to things based on their playing a part, assuming a function or being used in some situation".

A verb concept (or fact type) represents the notion of relations and is defined as "a concept that is the meaning of a verb phrase". A fact type can have one (characteristic), two (binary) or more fact type roles. 


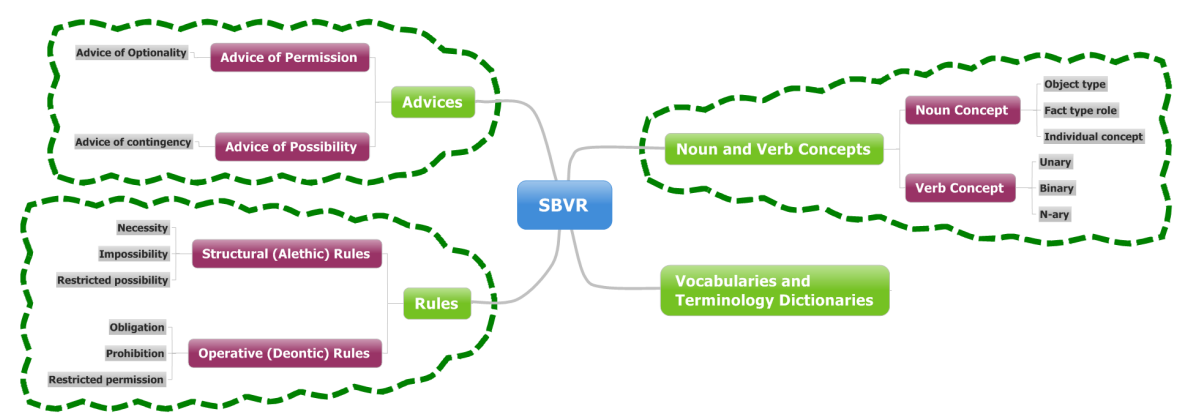

Fig. 1. SBVR overview

Business rules. The main types of rules defined in SBVR standard are structural business rules and operative business rules (See Figure 1). Structural (definitional) rules specify what the organization takes things to be, how do the members of the community agree on the understanding of the domain [5]. They define the characteristics of noun concepts and put constraints on verb concepts and can not be broken. Operative (behavioural) business rules are intended to describe the business processes in organization and can be either ignored or violated by people.

Conceptual model. An SBVR conceptual model $C M=\langle S, F\rangle$ is a structure intended to describe a business domain, where $S$ is a conceptual schema, declaring fact types and rules relevant to the business domain, and $F$ is a population of facts that conform to this schema. Business rules defined in the conceptual schema $S$ can be considered as high-level facts (i.e., facts about propositions) and play a role of constraints, which are used to impose restrictions concerning fact populations.

The SBVR standard provides means for formally expressing business facts and business rules in terms of fact types of pre-defined schema and certain logical operators, quantifiers, etc. These formal statements of rules may be transformed into logical formulations, which can in turn be used for exchange with other rules-based software tools. Such logical rule formulations are equivalent to formulae in 2-valued first-order predicate calculus with identity [19]. In addition to standard universal $(\forall)$ and existential $(\exists)$ quantifiers, for the sake of convenience, SBVR standard allows logical formulation to use some pre-defined [8] numeric quantifiers, such as at-most-one $\left(\exists^{0 . .1}\right)$, exactly- $n\left(\exists^{n}, n \geq 1\right)$ and others.

In order to express the structural or operational nature of a business rule, the corresponding rule formulation uses any of the basic alethic or deontic modalities. Structural rule formulations use alethic operators: $\square=i$ is necessary that and $\diamond=$ it is possible that; while operative rule formulations use deontic modal operators $\boldsymbol{O}=$ it is obligatory that, $\boldsymbol{P}=$ it is permitted that, as well as $\boldsymbol{F}=i t$ is forbidden that. 
Notations for business vocabulary and rules. There are several common means of expressing facts and business rules in SBVR, namely through statements, diagrams or any combination of those, each serving best for different purposes (16, [19, Annex C, Annex L]). While graphical notations are helpful for demonstrating how concepts are related, they are usually impractical when defining vocabularies or expressing rules. We use $r$ to denote a business rule in SBVR regardless the particular format in which it is written. For the sake of readability we will denote any necessity claim as $r_{\square}$, possibility claim as $r_{\diamond}$, obligation claim as $r_{O}$ and permission claim as $r_{P}$.

One of the most promising notations for SBVR is Object-Role Modeling (ORM2), which is a conceptual modeling approach combining both formal, textual specification language and formal graphical modeling language 9]. ORM2 specification language applies to mixfix predicates of any arity and contains predefined patterns covering a wide range of constraints typical for business domains. An example of a structural rule expressed as necessity statement in ORM2 specification language is the following:

$$
r=\text { Each visitor has at most one passport. }
$$

An example illustrating ORM2 graphical notation is introduced on Figure 2 .

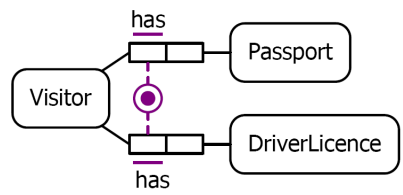

Fig. 2. Example of ORM2 diagram

The advantage of ORM2 over other notations is that it is a formal language per se, featuring rich expressive power, intelligibility, and semantic stability [11. There exist several translations from non-modal ORM2 expressions to standard logics, including translation to first-order logic ([10]) and some description logics ([14, [7]). We will hereafter denote by $\phi_{\hat{r}}$ a first-order representation of a nonmodal ORM2 expression $11 \hat{r}$ from a rule $r$. Similarly, we will denote by $\phi_{\hat{r}}^{D L}$ a description logic representation of a non-modal ORM2 expression $\hat{r}$.

Aforementioned existing translations to standard logics may be seen as attempts to provide a logical formalization for structural and operational rules. However, since they consider only the purely structural fragment of ORM2, they are not capable of providing consistency checks for a combined set of possibly interacting alethic and deontic business rules.

\footnotetext{
${ }^{1}$ Since the nature of business rules implies the absence of uncertainty, it means that the resulting first-order formulae will not contain free variables, i.e. will be closed formulae. Then an SBVR rule may be represented by an expression resulted from application of modalities and boolean connectives to a set of closed FOL formulae $\phi_{\hat{r_{i}}}$.
} 


\section{First-order deontic-alethic logic (FODAL)}

In this section we describe our attempt to provide logical foundations for SBVR by the means of defining a specific multi-modal logic. The basic formalisms we use to model business rule formulations are standard deontic logic $(\boldsymbol{S D L})$ and normal modal logic $\boldsymbol{S 4}$, which are both propositional modal logics. We then construct a first-order deontic-alethic logic (FODAL) - a multimodal logic, as a first-order extension of a combination of $\boldsymbol{S} \boldsymbol{D} \boldsymbol{L}$ and $\boldsymbol{S} \boldsymbol{4}$ to be able to express business constraints defined in SBVR. In order to construct the first-order extension for the combined logic we follow the procedure described in [6].

\subsection{Syntax}

The alphabet of FODAL contains the following symbols:

- a set of propositional connectives: $\neg, \wedge$.

- a universal quantifier: $\forall$ (for all).

- an infinite set $\mathcal{P}=\left\{P_{1}^{1}, P_{2}^{1}, \ldots, P_{1}^{2}, P_{2}^{2}, \ldots, P_{1}^{n}, P_{2}^{n}, \ldots\right\}$ of $n$-place relation symbols (also referred to as predicate symbols).

- an infinite set $\mathcal{V}=\left\{v_{1}, v_{2}, \ldots\right\}$ of variable symbols.

- modal operators: alethic $-\square$ (necessity) and deontic - $\boldsymbol{O}$ (obligation).

FODAL formulae. The formulae of FODAL are defined inductively in the following way:

- Every atomic formula is a formula.

- If $X$ is a formula, so is $\neg X$.

- If $X$ and $Y$ are formulae, then $X \wedge Y$ is a formula.

- If $X$ is a formula, then so are $\square X$ and $\boldsymbol{O} X$.

- If $X$ is a formula and $v$ is a variable, then $\forall v X$ is a formula.

The existential quantifier $(\exists)$ as well as other propositional connectives $(\vee, \rightarrow, \leftrightarrow)$ are defined as usual, while additional modal operators $(\diamond, \boldsymbol{P}, \boldsymbol{F})$ are defined in the following way:

$$
\diamond \phi \equiv \neg \square \neg \phi \quad \boldsymbol{P} \phi \equiv \neg \boldsymbol{O} \neg \phi \quad \boldsymbol{F} \phi \equiv \boldsymbol{O} \neg \phi
$$

A FODAL formula with no free variable occurrences is called a closed formula or a sentence. A modal sentence is a sentence whose main logical operator is a modal operator. An atomic modal sentence is a modal sentence which contains one and the only modal operator.

\subsection{Semantics}

Since SBVR itself interprets constraints in the context of possible worlds which correspond to states of the fact model (i.e. different fact populations), the choice of varying domain Kripke semantics is intuitively justified. Also, since SBVR rule formulations may includes two types of modalities: deontic and alethic, - we utilize the notion of two-layer Kripke frames with accessibility relations $R_{\boldsymbol{O}}$ and $R_{\square}$ respectively. 
Augmented frame. A varying domain augmented bimodal frame is a relational structure $\mathfrak{F}_{\text {var }}=\left\langle\mathcal{W}, R_{\boldsymbol{O}}, R_{\square}, \mathfrak{D}\right\rangle$, where $\left\langle\mathcal{W}, R_{\boldsymbol{O}}, R_{\square}\right\rangle$ is a two-layer Kripke frame, $\mathcal{W}$ is a non-empty set of worlds, $R_{(\cdot)}$ are binary relations on $\mathcal{W}$ and $\mathfrak{D}$ is a domain function mapping worlds of $\mathcal{W}$ to non-empty sets. A domain of a possible world $w$ is then denoted as $\mathfrak{D}(w)$ and a frame domain is defined as $\mathfrak{D}(\mathfrak{F})=\bigcup\left\{\mathfrak{D}\left(w_{i}\right) \mid w_{i} \in \mathcal{W}\right\}$.

In order to correctly capture the behavior and interaction of the alethic and deontic modal operators it is necessary to constrain the corresponding accessibility relations: the alethic accessibility is usually taken to be a reflexive and transitive relation $(\boldsymbol{S 4})$ [3], while the behavior of a deontic modality is classically considered to be captured by a serial relation $(\boldsymbol{K} \boldsymbol{D})[15$. We refer to the corresponding class of bimodal frames as $\boldsymbol{S} \mathbf{4} \otimes \boldsymbol{K} \boldsymbol{D}$-frames.

Moreover, since one of the objectives of formalization under development is to define the consistency of the set of business rules, it should also take into account the existing interaction between alethic and deontic modalities. The desired interaction can be verbalized as "Everything which is necessary is also obligatory" and then expressed as a following FODAL formula:

$$
\square X \rightarrow \boldsymbol{O} X
$$

It can be proved that the formula 2 defines a special subclass of $\boldsymbol{S} \otimes \boldsymbol{K} \boldsymbol{D}$-frames.

Theorem 1. The modal formula $\square X \rightarrow \boldsymbol{O} X$ defines the subclass of augmented bimodal $\boldsymbol{S} \mathbf{4} \otimes \boldsymbol{K} \boldsymbol{D}$-frames $\mathfrak{F}=\left\langle\mathcal{W}, R_{\boldsymbol{O}}, R_{\square}, \mathfrak{D}\right\rangle$ such that $R_{\boldsymbol{O}} \subseteq R_{\square}$, where $R_{\square}$ is a preorder and $R_{\boldsymbol{O}}$ is serial. We then call such frame a FODAL frame.

Proof: For the complete proof refer to [17.

Interpretation and model. An interpretation $\mathfrak{I}$ in a varying domain augmented frame $\mathfrak{F}_{\text {var }}=\left\langle\mathcal{W}, R_{\boldsymbol{O}}, R_{\square}, \mathfrak{D}\right\rangle$ is a function which assigns to each $m$ place relation symbol $P$ and to each possible world $w \in \mathcal{W}$ some $m$-place relation on the domain $\mathfrak{D}(w)$ of that world. $\mathfrak{I}$ can be also interpreted as a function that assigns to each possible world $w \in \mathcal{W}$ some first-order interpretation $\mathfrak{I}(w)$. An $\boldsymbol{F O D A L}$ varying domain first-order model is a structure $\mathfrak{M}=\left\langle\mathcal{W}, R_{\boldsymbol{O}}, R_{\square}, \mathfrak{D}, \mathfrak{I}\right\rangle$, where $\left\langle\mathcal{W}, R_{\boldsymbol{O}}, R_{\square}, \mathfrak{D}\right\rangle$ is a $\boldsymbol{F} \boldsymbol{O D} \boldsymbol{A} \boldsymbol{L}$ frame and $\mathfrak{I}$ is an interpretation in it.

Truth in a model. The satisfiability relation between $\boldsymbol{F O D A L}$ models and formulae is then defined in the usual way, using the notion of valuation which maps variables to elements of the domain.

Let $\mathfrak{M}=\left\langle\mathcal{W}, R_{\boldsymbol{O}}, R_{\square}, \mathfrak{D}, \mathfrak{I}\right\rangle$ be a $\boldsymbol{F O D A} \boldsymbol{L}$ model, $X, Y$ and $\Phi$ be FODAL formulae. Then for each possible world $w \in \mathcal{W}$ and each valuation $\sigma$ on $\mathfrak{D}(\mathfrak{M})$ the following holds:

- if $P$ is a m-place relation symbol, then $\mathfrak{M}, w \vDash_{\sigma} P\left(x_{1}, \ldots, x_{m}\right)$ if and only if $\left(\sigma\left(x_{1}\right), \ldots, \sigma\left(x_{m}\right)\right) \in \mathfrak{I}(P, w)$ or, equivalently, $\mathfrak{I}(w) \vDash_{\sigma}^{F O L} P\left(x_{1}, \ldots, x_{m}\right)$,

- $\mathfrak{M}, w \vDash_{\sigma} \neg X$ if and only if $\mathfrak{M}, w \nvdash_{\sigma} X$,

- $\mathfrak{M}, w \vDash_{\sigma} X \wedge Y$ if and only if $\mathfrak{M}, w \vDash_{\sigma} X$ and $\mathfrak{M}, w \vDash_{\sigma} Y$,

- $\mathfrak{M}, w \vDash_{\sigma} \forall x \Phi$ if and only if for every $x$-variant $\sigma^{\prime}$ of $\sigma$ at $w, \mathfrak{M}, v \vDash_{\sigma} \Phi$,

- $\mathfrak{M}, w \vDash_{\sigma} \exists x \Phi$ if and only if for some $x$-variant $\sigma^{\prime}$ of $\sigma$ at $w, \mathfrak{M}, v \vDash_{\sigma} \Phi$, 
- $\mathfrak{M}, w \vDash_{\sigma} \square X$ if and only if for every $v \in \mathcal{W}$ such that $w R_{\square} v, \mathfrak{M}, v \vDash_{\sigma} X$,

$-\mathfrak{M}, w \vDash_{\sigma} \diamond X$ if and only if for some $v \in \mathcal{W}$ such that $w R_{\square} v, \mathfrak{M}, v \vDash_{\sigma} X$,

- $\mathfrak{M}, w \vDash_{\sigma} \boldsymbol{O} X$ if and only if for every $v \in \mathcal{W}$ such that $w R_{\boldsymbol{O}} v, \mathfrak{M}, v \vDash_{\sigma} X$,

$-\mathfrak{M}, w \vDash_{\sigma} \boldsymbol{P} X$ if and only if for some $v \in \mathcal{W}$ such that $w R_{\boldsymbol{O}} v, \mathfrak{M}, v \vDash_{\sigma} X$.

\subsection{Axiomatization}

An FOD $A L$ axiom system for first-order alethic-deontic logic is defined following the approach presented in [6] and is obtained by combining the axiom systems for the propositional modal logics $\boldsymbol{S 4}$ and $\boldsymbol{K} \boldsymbol{D}$ and extending the resulting combination with additional axiom schemas and the axiom 9 reflecting desired interaction between alethic and deontic modalities.

Axioms. All the formulae of the following forms are taken as axioms.

(Tautologies $\boldsymbol{S 4}$ ) Any FOL substitution-instance of a theorem of $\boldsymbol{S 4}$

(Tautologies $\boldsymbol{K} \boldsymbol{D}$ ) Any FOL substitution-instance of a theorem of $\boldsymbol{K} \boldsymbol{D}$

(Vacuous $\forall$ ) $\forall x \phi \equiv \phi$, provided $x$ is not free in $\phi$

$(\forall$ Distributivity) $\quad \forall x(\phi \rightarrow \psi) \rightarrow(\forall x \phi \rightarrow \forall x \psi)$

$(\forall$ Permutation) $\forall x \forall y \phi \rightarrow \forall y \forall x \phi$

$(\forall$ Elimination) $\quad \forall y(\forall x \phi(x) \rightarrow \phi(y))$

(Necessary $\boldsymbol{O}) \quad \square \phi \rightarrow \boldsymbol{O} \phi$

Rules of inference.

$$
\begin{array}{llll}
\text { (Modus Ponens) } & \frac{\phi \phi \rightarrow \psi}{\psi} & \text { (Alethic Necessitation) } & \frac{\phi}{\square \phi} \\
\text { (Deontic Necessitation) } \frac{\phi}{\boldsymbol{O} \phi} & (\forall \text { Generalization) } & \frac{\phi}{\forall x \phi}
\end{array}
$$

Theorem 2. The FODAL axiom system is complete and sound with respect to the class of $\boldsymbol{F O D A L}$ frames.

Proof: For the complete proof refer to [17.

\section{Modeling SBVR vocabulary and rules with FODAL}

Given an SBVR conceptual schema $\mathcal{S}$ we define the following translation $\tau(\cdot)$ from elements of $\mathcal{S}$ to notions of first-order deontic-alethic logic:

- For each noun concept $A$ from $\mathcal{S}, \tau(A)$ is an unary predicate in FODAL.

- For each verb concept $R$ from $\mathcal{S}, \tau(R)$ is an $n$-ary predicate in FODAL $(n \geq 2)$.

Recall that an SBVR business rule may be represented by an expression resulted from application of modalities and boolean connectives to a set of closed firstorder formulae $\phi_{\hat{r_{i}}}$. Then for each SBVR rule $r$ from $\mathcal{S}$, its FODAL formalization $\tau(r)$ is defined inductively as follows: 
$-\tau(\hat{r})=\phi_{\hat{r}}$, where $\hat{r}$ is an non-modal SBVR expression and $\phi_{\hat{r}}$ is its first-order translation,

$-\tau(\neg r)=\neg \tau(r)$,

$-\tau\left(r_{1} \circ r_{2}\right)=\tau\left(r_{1}\right) \circ \tau\left(r_{2}\right), \circ \in\{\wedge, \vee, \rightarrow, \leftrightarrow\}$, where $r_{1}$ and $r_{2}$ are rule formulations,

$-\tau(\square \hat{r})=\square \tau(\hat{r})$ and $\tau(\boldsymbol{O} \hat{r})=\boldsymbol{O} \tau(\hat{r})$.

Example 1. Assume the following set of business rules, expressed in SBVR Structured English:

$\left(r_{1}\right)$ Each car rental is insured by exactly one credit card.

$\left(r_{2}\right)$ Each luxury car rental is a car rental.

$\left(r_{3}\right)$ It is obligatory that each luxury car rental is insured by at least two credit cards.

Then the corresponding FODAL formulas are the following:

$$
\begin{aligned}
\tau\left(r_{1}\right) & =\forall x \exists^{1} y(\operatorname{CarRental}(x) \wedge \operatorname{Insured}(x, y)), \\
\tau\left(r_{2}\right) & =\forall x(\operatorname{LuxuryCarRental}(x) \rightarrow \operatorname{CarRental}(x)), \\
\tau\left(r_{3}\right) & =\boldsymbol{O}\left(\forall x \exists^{\geq 2} y(\operatorname{LuxuryCarRental}(x) \wedge \operatorname{Insured}(x, y))\right) .
\end{aligned}
$$

While our FODAL formalization of SBVR rules provides logical mechanism supporting rule formulations with multiple occurrences of modalities, SBVR standard mostly focuses on normalized business constraints [19, p.108] that may be expressed by rule statements of the form of atomic modal sentences or by statements reducible to such a form via mechanisms provided by FODAL axiomatization. As a matter of fact, restricting the domain of interest only to such atomic modal rule formulations allows to obtain some useful results concerning satisfiability reduction and connection to standard logics, as shown in 17.

Hereafter we will only consider SBVR rules expressible in one of the following forms of atomic modal sentences:

$$
\square \phi \quad \nabla \phi \quad \boldsymbol{O} \phi \quad \boldsymbol{P} \phi
$$

where $\phi$ is any closed wff of first-order logic.

In the case of having negation in front of the modal operator, we assume application of the standard modal negation equivalences in order to obtain the basic form of the initial rule.

FODAL regulation. A FODAL regulation $\Sigma$ is a set of FODAL atomic modal sentences formalizing structural and operational rules of an SBVR conceptual schema $S$. We introduce the following designations:

$$
\begin{gathered}
\tau\left(r_{\square}\right)=\square \eta, \quad \tau\left(r_{\diamond}\right)=\diamond \pi, \\
\tau\left(r_{\boldsymbol{O}}\right)=\boldsymbol{O} \theta, \quad \tau\left(r_{\boldsymbol{P}}\right)=\boldsymbol{P} \rho, \\
\Sigma=\left\{\square \eta_{1}, \ldots, \square \eta_{k}, \diamond \pi_{1}, \ldots, \diamond \pi_{l}, \boldsymbol{O} \theta_{1}, \ldots, \boldsymbol{O} \theta_{m}, \boldsymbol{P} \rho_{1}, \ldots, \boldsymbol{P} \rho_{n}\right\} \\
\Sigma_{\wedge}=\bigwedge_{i=1}^{k} \square \eta_{i} \wedge \bigwedge_{i=1}^{l} \diamond \pi_{i} \wedge \bigwedge_{i=1}^{m} \boldsymbol{O} \theta_{i} \wedge \bigwedge_{i=1}^{n} \boldsymbol{P} \rho_{i}
\end{gathered}
$$

where every $\eta_{i}, \pi_{i}, \theta_{i}, \rho_{i}$ is a closed first-order logic formula. 


\section{Consistency of a set of business rules}

The final objective of the proposed formalization is to provide an automation solution with reasoning support for SBVR business modeling and business processes monitoring. It is well known that when reasoning about some particular universe of discourse, consistency is essential.

Assume a FODAL regulation $\Sigma$ representing a set of structural and operative business rules. The task of consistency check for $\Sigma$ is defined as procedure which analyzes the given set $\Sigma$ and decides whether none the rules contradict with each other, i.e. there is no formula $\psi$ such that $\Sigma \vdash \psi$ and $\Sigma \vdash \neg \psi$.

A FODAL regulation $\Sigma$ is called internally inconsistent when the specified constraints do not contradict each other when the system is populated.

We distinguish several types of inconsistency depending on types of modalities of rules involved. The set $\Sigma$ is called alethic inconsistent if it is inconsistent and the minimal inconsistent set $\Sigma_{\perp}$ contains formulae of only alethic nature, i.e. $\Sigma_{\perp} \subseteq \Sigma_{\square}$. The set $\Sigma$ is called deontic inconsistent if it is inconsistent and the minimal inconsistent set $\Sigma_{\perp}$ contains formulae of only deontic nature, i.e. $\Sigma_{\perp} \subseteq \Sigma_{\boldsymbol{O}}$. Otherwise, if $\Sigma_{\perp} \subseteq \Sigma_{\square} \cup \Sigma_{\boldsymbol{O}}$, the set $\Sigma$ is called cross inconsistent.

According to the completeness of the FODAL logic we have that $\Sigma \nvdash \psi$ if and only if there exists a FODAL model $\mathfrak{M}$ and a possible world $w$ in it, such that $\mathfrak{M}, w \vDash \Sigma \wedge \neg \psi$. Therefore, it is sufficient to state the satisfiability of the conjunction of all formulae of the set:

$$
\Sigma_{\wedge}=\bigwedge_{i=1}^{k} \square \eta_{i} \wedge \bigwedge_{i=1}^{l} \diamond \pi_{i} \wedge \bigwedge_{i=1}^{m} \boldsymbol{O} \theta_{i} \wedge \bigwedge_{i=1}^{n} \boldsymbol{P} \rho_{i}
$$

Bearing in mind the fact that the regulation $\Sigma$ may only contain FODAL atomic modal sentences and taking into account the properties of accessibility relations of the FODAL frame $\mathfrak{F}$, we can obtain the following result:

Theorem 3. A FODAL regulation $\Sigma_{\wedge}=\bigwedge_{i=1}^{k} \square \eta_{i} \wedge \bigwedge_{i=1}^{l} \diamond \pi_{i} \wedge \bigwedge_{i=1}^{m} \boldsymbol{O} \theta_{i} \wedge$ $\bigwedge_{i=1}^{n} \boldsymbol{P} \rho_{i}$ is FODAL-satisfiable if and only if each of the following formulae $\mathcal{N}, \mathcal{O}, \mathcal{Q}_{j}, \mathcal{P}_{j}$ is independently first-order satisfiable:

$$
\begin{aligned}
& \mathcal{N}=\bigwedge_{i=1}^{k} \eta_{i} \\
& \mathcal{O}=\bigwedge_{i=1}^{m} \theta_{i} \wedge \bigwedge_{i=1}^{k} \eta_{i} \\
& \mathcal{Q}_{j}=\pi_{j} \wedge \bigwedge_{i=1}^{k} \eta_{i}, \quad \forall j=\overrightarrow{1 \ldots l} \\
& \mathcal{P}_{j}=\rho_{j} \wedge \bigwedge_{i=1}^{m} \theta_{i} \wedge \bigwedge_{i=1}^{k} \eta_{i}, \quad \forall j=\overrightarrow{1 \ldots n}
\end{aligned}
$$

Proof: For the complete proof refer to [17. 
Observe that satisfiability of $\mathcal{N}$ follows naturally from satisfiability of any $\mathcal{Q}_{j}$. The same holds for $\mathcal{O}$ and $\mathcal{P}_{j}$ respectively. However, the satisfiability checks for $15 \mathrm{a}$ and $15 \mathrm{~b}$ should be examined explicitly, since $\Sigma$ may only contain necessity and obligation rules. Moreover, such definition allows to detect the actual source of unsatisfiability of the FODAL regulation $\Sigma$.

Modularity of the approach. It should be noted that the developed approach of satisfiability reduction possesses a property of modularity, i.e. it does not depend on the formalism behind the rule bodies $\eta_{i}, \theta_{i}, \pi_{i}$ and $\rho_{i}$, as long as formalism-specific satisfiability relation is provided.

\section{Reduction from FODAL to monomodal logic $Q K$}

As a matter of fact, the FODAL logic inherits the property of undecidability from both its component logics: standard predicate modal logics $\boldsymbol{Q S 4}$ and $\boldsymbol{Q K D}$ are undecidable [13. However, decidability results have been obtained for several well-studied fragments of quantified modal logics [20. This section defines a truth-preserving translation of atomic modal sentences of the FODAL logic into standard predicate modal logic $\boldsymbol{Q K}$, which allows to use those results.

Monomodal simulating pointed frame. Given a FODAL frame $\mathfrak{F}=\left\langle\mathcal{W}, R_{\boldsymbol{O}}, R_{\square}, \mathfrak{D}\right\rangle$ and a possible world $w_{0} \in \mathcal{W}$, a monomodal simulating pointed frame $\mathfrak{F}_{w_{0}}^{s}$ is defined as a tuple $\left\langle\mathcal{W}^{s}, R^{s}, \mathfrak{D}^{s}, w_{0}\right\rangle$, such that:

- $\mathcal{W}^{s}$ includes $w_{0}$ and all its deontic and alethic successors: $\mathcal{W}^{s}=\left\{w_{0}\right\} \cup\left\{v \mid\left(w_{0}, v\right) \in R_{O}\right\} \cup\left\{v \mid\left(w_{0}, v\right) \in R_{\square}\right\}=\mid$ since $R_{\boldsymbol{O}} \subseteq R_{\square}$ and $R_{\square}$ is reflexive $\mid=\left\{v \mid\left(w_{0}, v\right) \in R_{\square}\right\}$.

- $R^{s}=\left\{\left(w_{0}, v\right) \mid\left(w_{0}, v\right) \in R_{\square}\right\}$, and $\square^{s}, \searrow^{s}$ are modal operators associated with $R^{s}$.

- $\mathfrak{D}^{s}$ is a domain function on $\mathcal{W}^{s}$ such that $\mathfrak{D}^{s}(v)=\mathfrak{D}(v) \cup\left\{\pi^{\mathfrak{D}^{\mathfrak{s}}}\right\}, \forall v \in \mathcal{W}^{s}$, where $\pi^{\mathfrak{D}^{\mathfrak{S}}} \notin \mathfrak{D}$ is a new service domain symbol.

Since the definition of $R^{s}$ does not preserve specific properties of $R_{O}$ and $R_{\square}$, the resulting frame $\mathfrak{F}_{w_{0}}^{s}$ belongs neither to serial nor to transitive nor to reflexive class of frames and therefore can be classified as a $\mathbf{K}$-frame.

Monomodal translation. Given a FODAL regulation $\Sigma$ expressed as a conjunction of FODAL atomic modal sentences 14 a monomodal translation of regulation $\operatorname{MTR}\left(\Sigma_{\wedge}\right)$ is defined inductively as follows:

$$
\begin{aligned}
& \operatorname{MTR}(\phi) \quad=\phi \text {, where } \phi \text { is an objective FODAL formula, } \\
& \operatorname{MTR}\left(\phi_{1} \wedge \phi_{2}\right)=\operatorname{MTR}\left(\phi_{1}\right) \wedge M T R\left(\phi_{2}\right) \text {, where } \phi_{1} \text { and } \phi_{2} \text { are FODAL atomic } \\
& \text { modal sentences, } \\
& \operatorname{MTR}(\square \psi)=\square^{s} \operatorname{MTR}(\psi), \quad \operatorname{MTR}(\boldsymbol{O} \psi)=\square^{s}(\neg \Pi \rightarrow \operatorname{MTR}(\psi)), \\
& \operatorname{MTR}(\diamond \psi)=\diamond^{s}(\operatorname{MTR}(\psi) \wedge \Pi), \quad \operatorname{MTR}(\boldsymbol{P} \psi)=\diamond^{s}(\operatorname{MTR}(\psi) \wedge \neg \Pi),
\end{aligned}
$$

where $\psi$ is a objective FODAL formula and $\Pi$ is a 0 -place predicate symbol, i.e. propositional letter, encapsulating the nature of the original modality of the rules of possibility and permission. 
Simulated pointed model. Given a FODAL model $\mathfrak{M}=\langle\mathfrak{F}, \mathfrak{I}\rangle$ and a possible world $w_{0} \in \mathcal{W}$, a simulated pointed model $\mathfrak{M}_{w_{0}}^{s}$ is defined as a tuple $\left\langle\mathfrak{F}_{\mathfrak{w}_{\mathfrak{o}}}^{\mathfrak{s}}, \mathfrak{I}^{\mathfrak{s}}\right\rangle$ such that:

$-\mathfrak{F}_{w_{0}}^{s}=\left\langle\mathcal{W}^{s}, R^{s}, \mathfrak{D}^{s}, w_{0}\right\rangle$ is a monomodal simulating pointed frame for $\mathfrak{F}=$ $\left\langle\mathcal{W}, R_{\boldsymbol{O}}, R_{\square}, \mathfrak{D}\right\rangle$ and a possible world $w_{0} \in \mathcal{W}$,

- $\mathfrak{I}^{s}$ is a first-order interpretation on the frame $\mathfrak{F}_{w_{0}}^{s}$ such that:

- For each $v \in \mathcal{W}^{s}$ and for every $n$-place predicate $P, \mathfrak{I}^{s}(P, v)=\mathfrak{I}(P, v)$,

- For each $v \in \mathcal{W}^{s}$ such that $\left(w_{0}, v\right) \in R_{\boldsymbol{O}}, \mathfrak{I}^{s}(\Pi, v)=\varnothing$,

- For each $v \in \mathcal{W}^{s}$ such that $\left(w_{0}, v\right) \notin R_{\boldsymbol{O}}, \mathfrak{I}^{s}(\Pi, v)=\left\{\pi^{\mathfrak{D}^{\mathfrak{s}}}\right\}$.

We now state formally that the translation given above is truth-preserving with respect to varying domain semantics.

Theorem 4. For any FODAL regulation $\Sigma$, any FODAL model $\mathfrak{M}$ and any possible world $w_{0}$ of a model, we have that

$$
\begin{array}{r}
\mathfrak{M}, w_{0} \vDash \Sigma \quad \text { if and only if } \mathfrak{M}_{w_{0}}^{s}, w_{0} \vDash M T R(\Sigma), \\
\text { where } \mathfrak{M}_{w_{0}}^{s} \text { is a simulated pointed model for } \mathfrak{M} \text { and } w_{0} .
\end{array}
$$

Proof: For the complete proof refer to [17.

Therefore, the truth-preserving translation $M T R$ defined for FODAL regulations enables the transfer of decidability results from well-studied fragments of predicate modal logics (20], 1] ) to FODAL. In particular, the following fragments of FODAL logic are decidable:

- the set of atomic modal sentences with at most two variables,

- the set of monadic atomic modal sentences, all predicate symbols in which are at most unary,

- the set of atomic modal sentences, modal operators in which are applied to subformulas from the guarded fragment of first-order logic.

\section{Implementation of automated reasoning support tool}

\subsection{General description of the tool}

The ORM2 automated reasoning support tool is implemented in Java and includes a parser for ORM2 Formal Syntax [7, a set of Java classes representing the ORM2 knowledge database, a translator into an OWL2 ontology and a modal reasoning engine using HermiT or $\mathrm{FaCT}++$ as an underlying reasoner. The workflow diagram of the tool is depicted on Figure 3 . Currently, the tool provides the following functionality:

- Checking the consistency of a given ORM2 schema which may include both alethic (necessities and possibilities) and deontic (obligations and permissions) constraints. One of the advantages of the underlying approach is the straight-forward possibility to determine whether the inconsistency is caused purely by alethic or deontic constraints or by their combination. Additionally to the result of the consistency check, the tool prints out the list of concepts which are involved in conflicting constraints. 
- Translating a given ORM2 schema into OWL2 ontology which can then be saved in various formats for further use. However, this translation does not support modalities in their diversity and, therefore, takes into account only structural constraints (i.e. alethic rules).

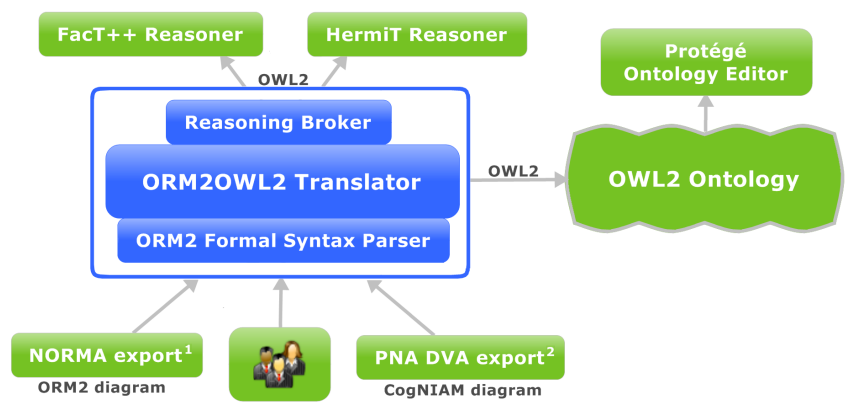

Fig. 3. Workflow Diagram

\footnotetext{
${ }^{1}$ Neumont ORM Architect for Visual Studio

${ }^{2}$ PNA Group Discovery and Validation Assistant
}

\subsection{Logical foundations of implementation}

The algorithm of the developed automated reasoning support tool relies on two fundamental results.

Firstly, it implements the procedure defined in [7 to translate a set of constraints from ORM2 Formal Syntax to $\mathcal{A L C} \mathcal{L I}$ description logic, which is in fact a fragment of OWL2. Since the implemented ORM2 reasoning procedure involves less expressive $\mathcal{A L C} \mathcal{Q I}$ logic as underlying formalism [17, it does not support reasoning about the following information about the ORM2 conceptual schema:

- frequency constraints on multiple roles,

- generalized subset constraints on relations,

- still supported: simple case of stand-alone roles,

- still supported: special case of contiguous full-set of roles;

- ring constraints (NB: drawback of mapping n-ary relations via reification).

The same information is lost by translating a given ORM2 schema into OWL2 ontology.

Secondly, in order to check the consistency of a set of business rules expressed in $\mathcal{A L C} \mathcal{L I}$-definable fragment of ORM2, we utilize the modularity of the approach defined in Section 5 and adapt the result of satisfiability reduction for the case of general description logic $D L$. The satisfiability relation for ORM2 is then provided by the semantic-preserved translation from ORM2 Formal Syntax to $\mathcal{A L C Q I}[7$. 
Theorem 5. A FODAL regulation $\Sigma=\left\{\square \eta_{1}, \ldots, \square \eta_{k}, \diamond \pi_{1}, \ldots, \diamond \pi_{l}, \boldsymbol{O} \theta_{1}, \ldots, \boldsymbol{O} \theta_{m}\right.$ $\left.\boldsymbol{P} \rho_{1}, \ldots, \boldsymbol{P} \rho_{n}\right\}$, expressed in $D L$-definable fragment of ORM2, is internally consistent if and only if each of the following description logic formulae $\mathcal{N}^{D L}, \mathcal{O}^{D L}$, $\mathcal{Q}_{j}^{D L}, \mathcal{P}_{j}^{D L}$ is independently satisfiable in $D L$ :

$$
\begin{array}{cc}
\mathcal{N}^{D L}=\prod_{i=1}^{k} \eta_{i} & \mathcal{O}^{D L}=\prod_{i=1}^{m} \theta_{i} \sqcap \prod_{i=1}^{k} \eta_{i} \\
\mathcal{Q}_{j}^{D L}=\pi_{j} \sqcap \prod_{i=1}^{k} \eta_{i}, & \mathcal{P}_{j}^{D L}=\rho_{j} \sqcap \prod_{i=1}^{m} \theta_{i} \sqcap \prod_{i=1}^{k} \eta_{i}, \quad \forall j=\overrightarrow{1 \ldots n}
\end{array}
$$

Thus, we can reduce the consistency of a given set of constraints to $\mathcal{A L C} \mathcal{Q I}$ satisfiability, which in turn can be interpreted as unsatisfiable concepts' check in resulting OWL2 ontology. Indeed, whenever a formula in $\mathcal{A L C} \mathcal{L I}$ is unsatisfiable, it means that the concept definition expressed by this formula contains a contradiction which prevents the concept from having a model, i.e. the concept is forced to not have any instances, hence is unsatisfiable.

\subsection{Usage of the tool}

In the following section we will demonstrate the functionality of the implemented tool by means of a real-life example of its usage. The graphical user interface of a tool is introduced on Figure 5 and contains controls which allow to select an input file, underlying reasoner, output file and output format for resulting ontology (if needed). The consistency check for an input ORM2 schema is performed after loading the input file and the result of the check is communicated by visual flag as well as by a detailed log in the corresponding window.

Checking the consistency of a given ORM2 schema. In order to illustrate the functionality of the consistency check we will consider the ORM2 schema obtained by merging two conceptual models (e.g. $A$ and $B$ ) and depicted on the Figure 4. This schema contains the following set of conflicting business rules:
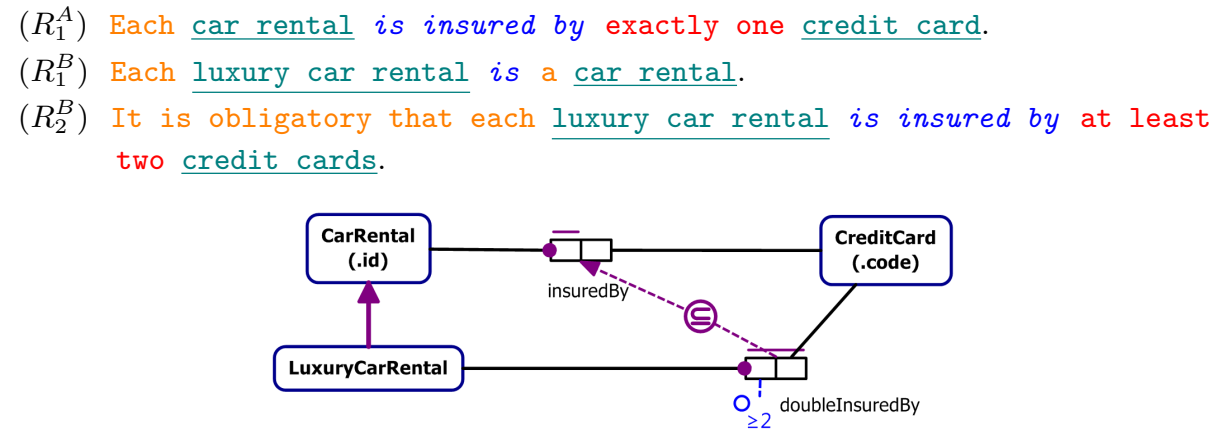

Fig. 4. Inconsistent ORM2 Schema

The given set of constraints can be fully expressed in $\mathcal{A L C Q \mathcal { I }}$ description logic, therefore we can use the developed reasoning support tool for consistency check. The schema on Figure 4 is internally inconsistent with respect to obligation $\left(R_{2}^{B}\right)$ since the latter clearly contradicts the structural constraint $\left(R_{1}^{A}\right)$ 
which, together with is- $a$ constraint on luxury car rental, simply does not support more than one credit card. Therefore, for any luxury car rental the obligatory cardinality constraint cannot be satisfied. The same conclusion is indeed inferred by the implemented tool on Figure 5.

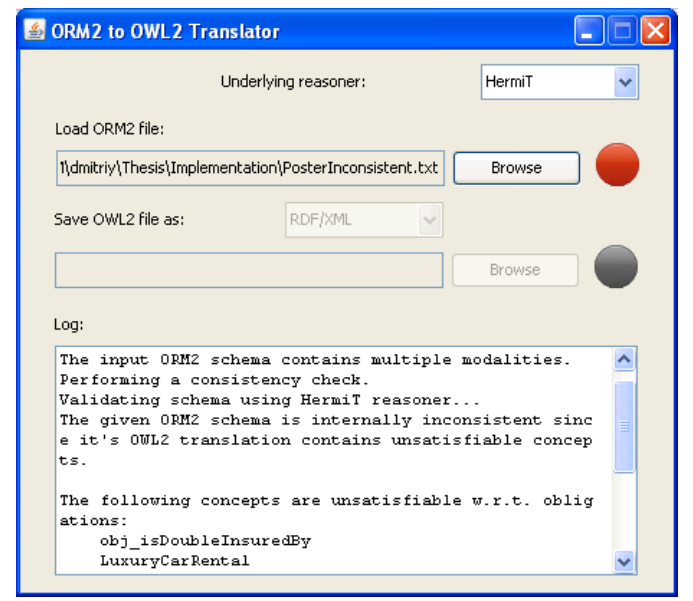

Fig. 5. The Graphical User Interface

\section{Conclusion}

In this paper we introduced a logical formalization of the Semantics of Business Vocabulary and Rules standard (SBVR) by defining a first-order deontic-alethic logic (FODAL) with its syntax, semantics and complete and sound axiomatization, that captures the semantics of and interaction between business rules.

We also showed that satisfiability in FODAL logic may be reduced to a standard first-order satisfiability for a class of formulas restricted to atomic modal sentences. Moreover, in order to establish a relationship with a standard logical formalism, we defined a truth-preserving translation from a fragment of bimodal FODAL into quantified monomodal logic $\boldsymbol{Q} \boldsymbol{K}$, that can be used to facilitate the transfer of decidability results from well-studied fragments of predicate modal logics to FODAL.

Finally we presented the ORM2 reasoning tool which provides an automated support for consistency checks of the conceptual model along with its translation to OWL2 ontology. The main functionality of the tool is a consistency check of a set of $\mathcal{A L C} \mathcal{L I}$-expressible deontic and alethic business rules. Another important task supported by the tool is translation of the aforementioned fragment of an ORM2 schema into an OWL2 ontology, which, however, does not support any modalities except necessity due to lack of notions representing deontic constraints in OWL2.

The future research in the field of logical formalization of SBVR aims to study the problem of entailment with respect to possible interaction of alethic and deontic modalities. Another future course of work includes defining an approach to translate an ORM2 schema with its alethic and deontic rules to SWRL or some other extension of OWL2. 


\section{References}

1. Hajnal Andréka, Johan Van Benthem, and István Németi. Modal languages and bounded fragments of predicate logic, 1996.

2. Donald E. Baisley, John Hall, and Donald Chapin. Semantic formulations in SBVR. In Rule Languages for Interoperability. W3C, 2005.

3. Patrick Blackburn, Maarten de Rijke, and Yde Venema. Modal Logic. Number 53 in Cambridge Tracts in Theoretical Computer Science. Cambridge University Press, UK, 2001.

4. Paolo Ceravolo, Cristiano Fugazza, and Marcello Leida. Modeling semantics of business rules. In Proceedings of the Inaugural IEEE International Conference On Digital Ecosystems and Technologies (IEEE-DEST), February 2007.

5. Donald Chapin. SBVR: What is now possible and why? Business Rules Journal, $9(3), 2008$.

6. Melvin Fitting and Richard L. Mendelsohn. First-order modal logic. Kluwer Academic Publishers, Norwell, MA, USA, 1999.

7. Enrico Franconi, Alessandro Mosca, and Dmitry Solomakhin. ORM2: Syntax and semantics. Internal report, KRDB Research Centre for Knowledge and Data, 2011.

8. Terry Halpin. A Logical Analysis of Information Systems: Static Aspects of the Data-oriented Perspective. PhD thesis, Department of Computer Science, University of Queensland, 1989.

9. Terry Halpin. Object-Role Modeling (ORM/NIAM). In Handbook on Architectures of Information Systems, pages 81-102. Springer-Verlag, 1998.

10. Terry Halpin and Tony Morgan. Information Modeling and Relational Databases. Morgan Kaufmann Publishers Inc., San Francisco, CA, USA, 2 edition, 2008.

11. Terry A. Halpin and Jan Pieter Wijbenga. FORML 2. In Ilia Bider, Terry A. Halpin, John Krogstie, Selmin Nurcan, Erik Proper, Rainer Schmidt, and Roland Ukor, editors, BMMDS/EMMSAD, volume 50 of Lecture Notes in Business Information Processing, pages 247-260. Springer, 2010.

12. Rami Hodrob and Mustafa Jarrar. Mapping ORM into OWL 2. In Proceedings of the 1st International Conference on Intelligent Semantic Web-Services and Applications, ISWSA '10, pages 9:1-9:7, New York, NY, USA, 2010. ACM.

13. G. E. Hughes and M. J. Cresswell. A New Introduction To Modal Logic. Routledge, 1996.

14. C. Maria Keet. Mapping the Object-Role Modeling language ORM2 into Description Logic language DLRifd. CoRR, abs/cs/0702089, 2007.

15. Paul McNamara. Deontic logic. In Edward N. Zalta, editor, The Stanford Encyclopedia of Philosophy. Fall 2010 edition, 2010.

16. Ronald G. Ross. Principles of the Business Rule Approach. Addison-Wesley Longman Publishing Co., Inc., Boston, MA, USA, 2003.

17. Dmitry Solomakhin. Logical Formalization of Semantic Business Vocabulary and Rules. MSc thesis, Faculty of Informatics, Vienna University of Technology, http: //media.obvsg.at/AC07810206-2001, 2011.

18. The Business Rules Group. Defining business rules. What are they really? Technical report, The Business Rules Group, 2001.

19. The Object Management Group. Semantics of Business Vocabulary and Business Rules (SBVR). Formal specification, v1.0, 2008.

20. Frank Wolter and Michael Zakharyaschev. Decidable fragments of first-order modal logics. J. Symb. Log., 66(3):1415-1438, 2001. 